\title{
Kluyveromyces lactis
}

National Cancer Institute

\section{Source}

National Cancer Institute. Kluyveromyces lactis. NCI Thesaurus. Code C127724.

A species of yeasts in the family Saccharomycetaceae and phylum Ascomycota with the ability to utilize lactose and convert it into lactic acid. The species is heterothallic, with a predominantly haploid life cycle. K. lactis has emerged as an important yeast species for research and industrial biotechnology. 